Veritas 日 Scientia

Vol. 9. $\mathrm{N}^{\circ} 1$

Enero - Junio del 2020

ISSN Edición Online: 2617-0639

https://doi.org/10.47796/ves.v9i1.283

\title{
EFECTOS DEL DUMPING EN LA COMPETITIVIDAD DE LAS EMPRESAS DE CONFECCIONES DE PRENDAS DE VESTIR DEL EMPORIO COMERCIAL DE GAMARRA, AÑO 2016
}

\section{DUMPING IN COMPETITIVENESS IN CLOTHING COMPANIES OF THE GAMARRA COMMERCIAL EMPORIO, YEAR 2016}

\author{
Elmer Reyes Trujillo ${ }^{1}$ \\ José Antonio Peña Rivera ${ }^{2}$
}

Aceptado: $16 / 02 / 2020$

Publicado online:17/07/2020

\begin{abstract}
RESUMEN
El presente trabajo de investigación tiene como objetivo determinar los efectos del dumping en la competitividad de las empresas de confecciones de prendas de vestir en el Emporio Comercial de Gamarra que iniciaron sus operaciones en el año 2016 y determinar cómo afecta el dumping en el crecimiento, posicionamiento y la utilidad de dichas empresas. Se partió de la hipótesis de que el dumping afecta negativamente en la competitividad de las empresas de confecciones de prendas de vestir; la desregulación normativa sobre el dumping y el inadecuado control que se pueda ejercer para el ingreso de este tipo de mercancías, fueron las causas para que algunos importadores encuentren la oportunidad para ingresar mercancías con precios dumping. El método de investigación fue descriptivo y explicativo en base a la información obtenida que nos permitió responder las interrogantes planteadas. Los resultados muestran que ambas variables tienen una estrecha relación positiva, ya que un alto riesgo del dumping generará una baja competitividad. Luego de la investigación se concluye que el ingreso al Perú de mercancías con precios por debajo del precio del producto similar en el país de fabricación destinado al consumo en el país exportador, afecta negativamente en el crecimiento empresarial del sector de confecciones de prendas de vestir, en el posicionamiento del mercado tanto interno como externo, así como en la utilidad que pueda percibir la empresa; obteniéndose como resultado el desempleo de los trabajadores, la reducción en la capacidad productiva, reducción en la capacidad competitiva, quiebra de las empresas de confecciones, cierre de fábricas del rubro afectado, y todo ello genera una desestabilidad económica en nuestro país; por tanto, se necesita una mayor participación del Estado en la
\end{abstract}

\footnotetext{
${ }_{1}^{1}$ Abogado, Magister en Gestión y Políticas Públicas, Especialista en Aduanas de la Superintendencia Nacional de Aduanas y Administración Tributaria. ereyest79@gmail.com, ereyest@sunat.gob.pe. ORCID: 0000-0002-4534-4371

2 Contador Público y Magister en Economía. Directivo en la Administración Pública del Perú. Especialista en tributación, auditoría y gestión pública. Académico de la Escuela de Postgrado de la Universidad Privada de Tacna. josea.pena@pucp.edu.pe. ORCID: 0000-0003-0985-0389
} 
regulación y el control de este tipo de productos, para de este modo proteger nuestra industria nacional.

Palabras claves: Dumping, Derechos Antidumping, Sector Textil y de Confecciones, Competitividad.

\begin{abstract}
The objective of this research work is to determine the effects of dumping on the competitiveness of garment apparel companies in the Gamarra Commercial Emporium that began operations in 2016 and determine how dumping affects the company's growth, positioning and income. It was hypothesized that dumping negatively affects the competitiveness of clothing apparel companies; the deregulation on dumping and the inadequate control that may be exercised for the entry of this type of merchandise, were the causes for some importers to find the opportunity to enter merchandise with dumping prices. The research method was descriptive and explanatory based on the information obtained that allowed us to answer the questions posed. The results show that both variables have a close positive relationship, since a high risk of dumping will generate a low competitiveness. After the investigation it is concluded that the entry into Peru of goods with prices below the price of the similar product in the country of manufacture destined for consumption in the exporting country, negatively affects the business growth of the apparel sector, in the positioning of the internal and external market, as well as in the utility that the company can perceive; Obtaining as result the unemployment of the workers, the reduction in the productive capacity, reduction in the competitive capacity, bankruptcy of the garment companies, closing factories of the affected item, and all this generates an economic instability in our country; Therefore, a greater participation of the State in the regulation and control of this type of products is needed, in order to protect our national industry.
\end{abstract}

Keywords: Dumping, Antidumping Rights, Textile and Clothing Sector, Competitiveness.

\title{
INTRODUCCIÓN
}

Una de las medidas comerciales correctivas implementadas por la Organización Mundial del Comercio (OMC), la más importante y la más utilizada por los países miembros, son las medidas antidumping. Entre los países que más utilizan estos mecanismos de protección contra las distorsiones comerciales en el comercio internacional son Estados Unidos, India, China y Australia, quienes las utilizan intensivamente. ¿La razón? Si nos detenemos a observar, nos damos cuenta que precisamente son aquellos países con mayor grado de desarrollo, quienes utilizan estos mecanismos para proteger su industria nacional, ante el peligro inminente de que sean afectados gravemente por la importación de productos similares, y 
generándose una competencia desleal, tanto al interior de sus mercados, como en el comercio internacional. Nuestro país, como miembro de la OMC, frente a estas prácticas comerciales desleales, ha hecho poco o nada por nuestra industria nacional, generándose una situación de riesgo y peligro, que en muchos casos llega al cierre de fábricas, con el consecuente despido de trabajadores y en un futuro cercano con una grave afectación a los consumidores nacionales, por un inminente monopolio del importador que ingresó productos a nuestro país con precios dumping. Este trabajo de investigación se encamina a coadyuvar y poner como una señal de alerta ya que se estaría afectando seriamente la competitividad de la industria de confecciones de prendas de vestir, quienes requieren de una mayor participación del Estado a fin de contrarrestar los efectos que pueden ser desastrosos, por el ingreso de prendas de vestir con precios dumping. Para su desarrollo se ha delimitado a las empresas de confecciones que fueron creadas en el año 2016 en el emporio comercial de Gamarra, resaltando su importancia ya que en la medida que protejamos nuestra industria nacional y pueda fortalecerse y convertirse en industrias cada vez más competitivas, se reflejará en el crecimiento económico de nuestro país.

El crecimiento económico constante en estos últimos años de los países orientales, y de los países conocidos como los BRICS (Brasil, Rusia, India, China, Sudáfrica), además de la recuperación económica mundial en estos dos últimos años, conforme a las fuentes del BM y el FMI, se aceleró del 3\% en el año 2017 al 3.1\% en el año 2018, gracias a una recuperación cíclica generalizada, en un mundo cada vez más globalizado, ha conllevado a que éstos tengan la necesidad de adquirir productos tales como insumos o materias primas para seguir creciendo, la cual ha sido satisfecha por las Economías de Mercados Emergentes y en Desarrollo (EMED).

En este contexto, también nuestro país en estos últimos años, ha visto incrementar su crecimiento económico; llegando en muchos casos a ser líder en crecimiento a nivel regional, como consecuencia de la llegada de inversiones nuevas y del incremento de las exportaciones, traduciéndose ello en una Balanza Comercial Favorable, lo que ha sido interrumpido únicamente en el periodo del 2013 al 2014, teniéndose un crecimiento económico por debajo de lo previsto. Según Reporte de Inflación: Panorama Actual y Proyecciones Macroeconómicas 2018 - 2019, publicado por el BCRP la proyección de crecimiento económico para el año 2018 es de $4 \%$ mientras que para el año 2019 es de 4.2\%. El Perú como país miembro de la OMC se encuentra facultada para poder corregir estas distorsiones en el mercado por el ingreso de mercancías con precios dumping; lo cual mediante Resolución № 297-2013-CFD/INDECOPI se hizo para el caso específico de las prendas de vestir en el periodo de 23 de diciembre de 2013 hasta el 07 de junio del 2015, fecha en que fue revocada mediante la Resolución № 02932015/SDC-INDECOPI (...). Según el Comité Textil de la Sociedad Nacional de Industrias, para el periodo de enero - marzo del 2018, el $71.3 \%$ en valor CIF de las prendas de vestir ingresaron desde la República Popular de China. Uno de los mecanismos de protección que nos proporciona la OMC, y que a su vez es muy eficaz para combatir la competencia desleal, es precisamente las medidas antidumping, herramienta que muchos países lo utilizan de manera efectiva para proteger su industria y corregir estas distorsiones en el mercado mundial. En México respecto a la evolución de las disposiciones antidumping en dicho país, (Barney Cruz, 2007), sostiene que del artículo VI del GATT y las diferentes rondas de negociación que fueron determinantes para la regulación de la materia, pasando por el Código Antidumping del GATT de 1967, el de 1979 y el de 1994. Según Cure Franco (2013) del análisis y comparación de los puntos relevantes en materia de los derechos antidumping y su aplicación por parte de la 
autoridad competente en Colombia con respecto a Estados Unidos y la Unión Europea, es posible concluir que hay elementos que le restan eficacia al mecanismo. Gutierrez Solsona (1994) realizó un trabajo para demostrar que las medidas antidumping, que hoy en día son tan utilizadas por la Unión Europea y por los Estados Unidos de América, tienen exclusivamente un fin proteccionista.

Conforme al trabajo de investigación de Abad Puelles (2002), el antidumping ha sido, y es usado, como una manera de proteccionismo hacia las industrias nacionales. (Rodríguez Fernández, 1998) propone la instauración de un sistema basado en la expedición de un denominado "certificado fair trade". En argentina (Zenere, 2013) se utilizan los mecanismos antidumping para combatir la competencia desleal en el comercio internacional y proteger la producción nacional; la diferencia radica en que Argentina hace un uso intensivo de este mecanismo, ubicándose a decir del autor entre los 10 países del orbe que más utilizan el procedimiento de investigación para la aplicación de las medidas antidumping; situación que nuestro país debería imitar. Darchuk, Milano, \& Curi (1999) concluye que, a nivel teórico, las medidas antidumping no eliminan las distorsiones de la economía mundial inherentes a los comportamientos de dumping. Nosotros consideramos que efectivamente el trato entre los países miembros y no miembros del Acuerdo debe ser diferenciado tanto en el aspecto arancelario, como en la aplicación de los derechos antidumping; tal como lo establece la norma MERCOSUR/CMC/DEC № 11/97. Crespo (1997) concluye que, aunque se evidencia cierta tendencia a regular y disminuir el perfil de las medidas para enfrentar el dumping, se pudiera esperar que en el mediano plazo las normativas antidumping continuarán ocupando un papel determinante en el desarrollo comercial entre las naciones. Montuschi (1993) considera que durante mucho tiempo el dumping ha sido considerado como un problema en el comercio internacional, una práctica desleal que se origina en la presencia de discriminación de precios en los mercados que se debe a la falta de operatividad de las fuerzas de la competencia. (Harrison, 2010) en su artículo brinda una breve, pero detallada introducción referida a los fenómenos del Dumping y subvenciones en el mercado australiano. Quispe Medina (2017) determinó tres modalidades no previstas en la legislación nacional: importar producto afecto con modificaciones o alteraciones menores, declarar que dicho producto proviene de un tercer país no afecto e importar el producto alterando los canales de comercialización; al respecto las normas administrativas, se encuentra regulada como una de las modalidades del delito de defraudación de rentas de aduana. Cabrera Marín \& Rodríguez Mercado,(2016) concluyen afirmando que el Tratado de Libre comercio suscrito con China incide favorablemente en la variación de las importaciones y exportaciones.(Castellares Añazco, 2016) refiere que las firmas más productivas mejoraron la calidad de sus productos para diferenciarse de las prendas chinas, mientras que las firmas menos productivas, que no fueron capaces de mejorar su calidad, optaron por reducir precios durante los años 20012007. Sobre la insuficiencia normativa de los derechos antidumping. Para Ramírez Estrella, (2018) la normativa nacional resulta insuficiente para garantizar la efectividad en la aplicación de los derechos antidumping. Según Díaz Hinostroza (2016), concluye que existe una relación significativa entre la aplicación de los derechos Antidumping y la Rentabilidad en las empresas textiles.

\section{OBJETIVOS}

Determinar cómo afecta el dumping en el crecimiento, posicionamiento y utilidad de las empresas de confecciones de prendas de vestir del Emporio Comercial de Gamarra, año 2016. 


\section{METODOLOGÍA}

Investigación básica no experimental, descriptiva y transversal. La muestra está conformada por 60 empresas del Emporio Comercial de Gamarra de la ciudad de Lima que iniciaron sus operaciones en el año 2016. Se utilizó la prueba estadística Modelo de Regresión Lineal y la prueba ANOVA, los cuales miden la causalidad entre las variables independiente y dependiente.

\section{RESULTADOS}

De las 60 empresas, 41 empresas consideran a la oportunidad para el dumping como de alto riesgo, lo cual representa el $68.3 \%$ de los encuestados, mientras que 19 empresas consideran de mediano riesgo, representando el $31.7 \%$ de las empresas encuestadas. De lo enunciado se puede determinar que el indicador oportunidad para el dumping tiene un alto riesgo en la importación de prendas de vestir que ingresan con precios dumping, representando el $68.3 \%$ de las empresas encuestadas, lo cual se puede considerar como altamente significativo.

Para determinar la técnica estadística a aplicar, se tomó en cuenta el tipo de variables a procesar, siendo ambas variables "Importación de prendas de vestir con precios dumping" y "Competitividad de las empresas de confecciones de prendas de vestir", de tipo ordinal, el estadístico seleccionado fue el Modelo de Regresión Lineal.

\begin{tabular}{llrrrr}
\hline Modelo & $\mathrm{R}$ & R cuadrado & $\begin{array}{c}\text { R cuadrado } \\
\text { ajustado }\end{array}$ & $\begin{array}{c}\text { Error estándar de la } \\
\text { estimación }\end{array}$ \\
\hline 1 &, $436^{\mathrm{a}}$ &, 190 & & 176 & 10,12746 \\
\hline
\end{tabular}

a. Predictores: (Constante), Dumping

Se puede observar un grado de correlación de 0,436 el cual representa una moderada correlación entre ambas variables. Asimismo, el R cuadrado o coeficiente de determinación es 0,19 lo cual significa que la variable independiente "Importación de prendas de vestir con precios dumping" explica el $19 \%$ de la variación observada en la variable dependiente "Competitividad de las empresas de confecciones de prendas de vestir".

\begin{tabular}{|c|c|c|c|c|c|c|}
\hline Modelo & & $\begin{array}{l}\text { Suma de } \\
\text { cuadrados }\end{array}$ & $\mathrm{gl}$ & Media cuadrática & $\mathrm{F}$ & Sig. \\
\hline \multirow[t]{3}{*}{1} & Regresión & 1394,858 & 1 & 1394,858 & 13,600 &, $001^{\mathrm{b}}$ \\
\hline & Residuo & 5948,792 & 58 & 102,565 & & \\
\hline & Total & 7343.650 & 59 & & & \\
\hline
\end{tabular}

a. Variable dependiente: Competitividad

b. Predictores: (Constante), Dumping

La prueba $\mathrm{F}$ de Fisher arrojó un valor de 13,60 el cual es mayor que el $\mathrm{F}$ de tabla para un nivel de significancia del $5 \%$. Asimismo, se puede verificar que el $p$ - valor es 0,00 (menor que $5 \%$ ) lo cual significa que la prueba ANOVA del modelo es significativa, lo que quiere decir que el modelo es válido a un nivel de confianza del $95 \%$.

\begin{tabular}{|c|c|c|c|c|c|c|}
\hline \multirow{2}{*}{\multicolumn{2}{|c|}{ Modelo }} & \multicolumn{2}{|c|}{ Coeficientes no estandarizados } & $\begin{array}{c}\text { Coeficientes } \\
\text { estandarizados }\end{array}$ & \multirow[b]{2}{*}{$\mathrm{t}$} & \multirow[b]{2}{*}{ Sig. } \\
\hline & & B & Error estándar & Beta & & \\
\hline \multirow[t]{2}{*}{1} & (Constante) & 22,438 & 7,227 & & 3,105 & ,003 \\
\hline & Dumping & ,464 & ,126 & ,436 & 3,688 & ,001 \\
\hline
\end{tabular}

a. Variable dependiente: Competitividad 


\section{DISCUSIÓN}

En el trabajo de investigación se encontró que existe una relación considerable entre "Importación de prendas de vestir con precios dumping" y "Competitividad de las empresas de confecciones de prendas de vestir"; ya que se ha evidenciado con los resultados obtenidos de la presente investigación que existe un alto riesgo de importaciones de prendas de vestir con precios dumping lo cual genera una baja competitividad en las empresas de confecciones de prendas de vestir. Tal es así que se ha demostrado que un alto riesgo en la importación de prendas de vestir con precios dumping genera un bajo crecimiento empresarial, un bajo posicionamiento de mercado de las empresas de confecciones y una baja utilidad del citado sector productivo. Ello se explica ya que, al ingresar este tipo de mercancías con precios por debajo del costo de fabricación de estas prendas de vestir, e inundar el mercado nacional con prendas de vestir importadas de China, a un precio tan bajo, lo que se hace es afectar negativamente los indicadores de competitividad empresarial detallados; consecuentemente, al afectar los indicadores de competitividad, se está afectando la competitividad de las empresas de confecciones de prendas de vestir.

En la oportunidad para el dumping se puede apreciar que tiene un alto riesgo en la importación de prendas de vestir con precios dumping, representando el $68.3 \%$. En cuanto a la regulación del dumping se determinó que tiene también un alto riesgo en la importación de prendas de vestir con precios dumping, representando el $61.7 \%$. En ambos casos, se pueden considerar como altamente significativo, que van a influir negativamente en la competitividad de las empresas de confecciones. En relación a los controles para el dumping tiene un mediano riesgo, representando el $60 \%$, lo cual se puede considerar como medianamente significativo y que también van a influir negativamente en la competitividad de las empresas de confecciones.

La razón de esta influencia negativa en la competitividad es que una desregulación normativa del dumping, así como los controles inadecuados que las autoridades nacionales puedan ejercer en el tratamiento de mercancías con precios dumping, va a incrementar el ingreso de prendas de vestir con precios dumping, lo que a su vez va a mermar la competitividad de las empresas del rubro de confecciones.

Por otro lado, el crecimiento empresarial, posicionamiento de mercado y la utilidad de las empresas, tienen un nivel de mediana competitividad, contando cada indicador con el $61.7 \%$, el $56.7 \%$ y el $80 \%$ respectivamente.

La razón, es que como ya se dijo, se ha determinado que existe una relación considerable entre "Importación de prendas de vestir con precios dumping" y el "Crecimiento empresarial", el "posicionamiento de mercado" y la "Utilidad de las empresas", lo cual implica que un alto riesgo de dumping generará un bajo crecimiento empresarial, bajo posicionamiento de mercado y una baja utilidad de las empresas.

Sobre los efectos del dumping en la competitividad de las empresas de confecciones de Gamarra coincidimos con las conclusiones de (Shupingahua Soria, 2017) en que es necesario una mayor participación del Estado en el crecimiento de las empresas, que el incremento de las importaciones con precios dumping generará desempleo, la práctica del dumping en la importación de confecciones chinas generó consecuencias negativas en la producción de prendas de vestir, y que las empresas por estas prácticas dumping generó menores utilidades; 
sin embargo la diferencia con nuestra investigación radica mientras que el $48.3 \%$ de los empresarios están de acuerdo sobre una mayor participación del Estado, y que las prácticas desleales en el comercio exterior generará desempleo; en su trabajo sostiene que el $100 \%$ de los empresarios consideran que no se sienten protegidos por el Estado y que el dumping inducirá a la informalidad y el desempleo.

En cuanto al trabajo de investigación de (Díaz Hinostroza, 2016) podemos apreciar que existe una similitud con el nuestro, ya que mediante este trabajo el autor llega a la conclusión que existe una relación significativa entre la aplicación de los derechos antidumping y la rentabilidad en las empresas textiles del distrito de La Victoria. La diferencia radica en que Díaz Hinostroza utilizó el sistema Dupont, creado por Donaldson Brown para llegar a su conclusión; es decir, examinó los estados financieros de las 40 empresas encuestadas y evaluó su margen de utilidad neta. Sin embargo, nosotros además de sostener que existe una relación significativa, concluimos que la relación es directamente proporcional entre la aplicación de los derechos antidumping y la rentabilidad de las empresas de confecciones, e inversamente proporcional entre la importación de prendas de vestir con precios dumping y la rentabilidad de las referidas empresas.

Quispe Medina (2017), en su investigación descriptiva determinó los efectos económicos y comerciales que ocurrieron en la industria nacional por actos de elusión de derechos antidumping o compensatorios bajo modalidades no previstas en la legislación nacional, como la de a) importar el producto afecto con modificaciones o alteraciones menores; b) declarar que dicho producto proviene de un tercer país no afecto; o c) importar el producto alterando los canales de comercialización en el país afecto. Como resultado de dicha evaluación se constató que entre los años 2003 y 2016, se eludió en el Perú el pago de los derechos antidumping aplicados a los tres productos antes referidos, bajo modalidades no previstas en la legislación nacional.

Coincidimos con la conclusión del autor sobre la desregulación normativa en las prácticas dumping; sin embargo, debemos aclarar que la desregulación normativa se da a nivel específico, es decir por la ausencia de estudios de casos para cada variedad de mercancía en concreto, como por ejemplo el caso de las prendas de vestir, dicha acción le compete exclusivamente a INDECOPI; en relación a las tres modalidades de elusión de los derechos antidumping, sostenemos que no existe la necesidad de incluir en las normas administrativas, ya que éste se encuentra regulada como una de las modalidades del delito aduanero de defraudación de rentas de aduana, previsto en el artículo 6으 de la Ley de Delitos Aduaneros, Ley № 28008, siendo sancionable penalmente. En cuanto a los efectos económicos y comerciales adversos que el autor señala, consideramos que uno de los requisitos para poder aplicarse los derechos antidumping en la importación de un producto determinado, es precisamente que exista un efecto adverso; es decir como señala la OMC y la legislación nacional, debe existir un daño en la rama de producción nacional; en caso contrario no existe ninguna razón para que pueda aplicarse los derechos antidumping.

El dumping afecta negativamente la competitividad de las empresas de confecciones de prendas de vestir del Emporio Comercial de Gamarra, según los resultados obtenidos de la encuesta realizada a dichos empresarios; no obstante, además del dumping existen otros factores que influyen negativamente en la competitividad tales como los recursos tecnológicos, la calidad, el capital humano, que también van a influir para que la competitividad de una empresa del sector. 
Se ha determinado que el dumping limita el crecimiento de las empresas de confecciones de prendas de vestir del Emporio Comercial, según los resultados obtenidos de la encuesta realizada a los empresarios de Gamarra, se puede apreciar que la relación del dumping con el crecimiento empresarial traen como consecuencia la reducción de la capacidad productiva de las empresas, lo cual genera la reducción del volumen de su producción; no obstante además del dumping existen otros factores que influyen negativamente en la competitividad de estas empresas, tales como la motivación de sus trabajadores, la estructura empresarial y como factores externos se puede citar los clientes, los proveedores, que también van a influir para que el crecimiento empresarial del sector confecciones de Gamarra pueda ser afectada.

Se ha verificado que el dumping reduce el posicionamiento y utilidad de mercado de las empresas de confecciones de prendas de vestir del Emporio Comercial de Gamarra, según los resultados obtenidos de la encuesta realizada a los empresarios de Gamarra, esto debido a que el ingreso de prendas de vestir con precios dumping genera una competencia desleal en el mercado interno de nuestro país, evitando que las empresas de confecciones de prendas de vestir puedan expandirse en el mercado nacional como en el extranjero; sin embargo, además del dumping existen otros factores que influyen negativamente en el posicionamiento de mercado de estas empresas, tales como la diferenciación con otras empresas del ramo, los beneficios relevantes para el consumidor, la marca, el prestigio comercial que también van a influir para que el posicionamiento de mercado del sector confecciones de Gamarra pueda ser afectada favorable o desfavorablemente.

Se ha verificado que no existe una regulación específica que permita corregir las distorsiones en el comercio exterior cuando ingresan a nuestro país prendas de vestir con precios dumping. Según la encuesta realizada a empresarios de confecciones, la desregulación normativa del ingreso de mercancías a nuestro país con precios dumping es considerada como una práctica de alto riesgo que distorsiona el mercado internacional y afecta gravemente el sector productivo involucrado.

\section{REFERENCIAS BIBLIOGRÁFICAS}

Abad Puelles, J. (11 de Noviembre de 2002). Impacto de las políticas antidumping en el proceso de integración de la Comunidad Andina. (Tesis de maestría, Universidad Andina Simón Bolivar). Ecuador. Obtenido de http://repositorionew.uasb.edu.ec/bitstream/10644/2442/1/T-0227MRI-Abad-Impacto.pdf

Artículo VI OMC. (1994). ACUERDO RELATIVO A LA APLICACIÓN DEL ARTíCULO VI DEL ACUERDO GENERAL SOBRE ARANCELES ADUANEROS Y COMERCIO DE 1994. Marrakech. Obtenido de https://ecampus.wto.org/admin/files/Course_767/Module_827/ModuleDocuments/19adp.pdf

Artículo VII de OMC. (1994). Acuerdo Relativo a la Aplicación del Artículo VII del Acuerdo General sobre Aranceles Aduaneros y Comercio de 1994. Marrakech. Obtenido de https://www.wto.org/spanish/tratop_s/cusval_s/cusval_info_s.htm

Barney Cruz, Ó. (Mayo - Agosto de 2007). Antecedentes del sistema contra prácticas desleales de comercio en México. La evolución de las disposiciones antidumping. 40. México D.F., México: Bol. Mex. Der. Obtenido de http://www.scielo.org.mx/scielo.php?pid=S0041$86332007000200005 \&$ script $=$ sci_arttext

Cabrera Marín, W., \& Rodríguez Mercado, N. W. (Febrero de 2016). Tratado de libre comercio entre Perú y China y su incidencia en la variación de las importaciones y exportaciones en el sector textil peruano: análisis antes y después de la firma del TLC, 2008 - 2014. (Tesis de grado, 
Universidad Privada Antonio Guillermo Urrelo), 174. Cajamarca, Perú: Universidad Privada Antonio Guillermo Urrelo. Obtenido de http://repositorio.upagu.edu.pe/handle/UPAGU/88

Cámara de Comercio de España. (s.f.). Innovación, Digitalización y Competitividad. Obtenido de Cámara de Comercio de España: https://www.camara.es/innovacion-y-competitividad/comoser-competitivo

Castellares Añazco, R. (Setiembre de 2016). Productividad en el Perú: medición, determinantes e implicancias. Lima, Perú. Obtenido de http://repositorio.up.edu.pe/handle/11354/1083

Crespo, R. J. (Diciembre de 1997). Dumping, Antidumping y Fundamentos del Antidumping. Bristol, Inglaterra. Obtenido de http://www.efm.bris.ac.uk/ecrc/Dumping.pdf

Cure Franco, K. (2013). Análisis Comparado Del Procedimiento Y La Aplicación De Derechos Antidumping. Colombia, Estados Unidos De America Y La Unión Europea. (Trabajo de grado, Pontificia Universidad Javeriana), 117. Bogotá, Colombia: Pontificia Universidad Javeriana. Obtenido https://repository.javeriana.edu.co/bitstream/handle/10554/10055/CureFrancoKarlaMargarit a2013.pdf?sequence $=1$

Darchuk, A., Milano, J., \& Curi, G. (1999). "Tratamiento de las prácticas de dumping y subvenciones en los procesos de integración, en especial el Mercosur". Buenos Aires, Argentina. Obtenido de http://aaep.org.ar/espa/anales/pdf_99/darchuk_milano_curi.pdf

Decisión 283 CAN. (21 de marzo de 1991). Normas para prevenir o corregir las Distorsiones en la competencia generadas por Prácticas Dumping o Subsidios. Lima, Lima, Perú. Obtenido de https://idatd.cepal.org/Normativas/CAN/Espanol/Decision_283-

Normas_para_prevenir_o_corregir_distorsiones_en_la_competencia.pdf

Decreto 210. (03 de Febrero de 2003). Objetivos y Estructura Orgánica del Ministerio de Comercio, Industria y Turismo. Diario Oficial de Colombia. Bogotá, Colombia. Obtenido de http://www.suin-juriscol.gov.co/viewDocument.asp?ruta=Decretos/1845734

Decreto Legislativo 1053. (27 de Junio de 2008). Ley General de Aduanas. El Peruano. Lima, Perú. Obtenido de http://www.sunat.gob.pe/legislacion/procedim/normasadua/gja-03.htm

Díaz Hinostroza, N. E. (2016). Derechos antidumping y su relación con la rentabilidad de las empresas textiles del distrito de la Victoria en el año 2015. (Tesis de grado, Universidad César Vallejo). Lima, Perú. Obtenido de http://repositorio.ucv.edu.pe/handle/UCV/2930

Espinosa, R. (15 de Seteimbre de 2014). Roberto Espinosa.es. Posicionamiento de marca, la batalla por tu mente. Obtenido de https://robertoespinosa.es/2014/09/15/posicionamiento-de-marcabatalla-por-mente/

Gianella Carbajal, R. G. (2017). Gestión del Talento Humano y Productividad Laboral en las Áreas de Enfermería y Obstetricia de una Red de Salud del Sur, 2016. Lima, Lima, Perú. Obtenido de http://repositorio.ucv.edu.pe/bitstream/handle/UCV/8641/Gianella_CRG.pdf?sequence=1\&is Allowed=y

Gutierrez Solsona, F. (1994). Medidas antidumping. Instrumento del neoproteccionismo. Madrid, España. Obtenido de https://econpapers.repec.org/paper/ucmdoctra/94-34.htm

Harrison, M. (17 de Febrero de 2010). Derechos Antidumping y Compensatorios en Australia La Aplicación de los Acuerdos de la OMC sobre el Dumping y Subsidios en Virtud de la Legislación Australiana. (PUCP, Ed.) Derecho \& Sociedad, 33, 163 - 170. Obtenido de http://revistas.pucp.edu.pe/index.php/derechoysociedad/article/viewFile/17467/17746

Instituto Naciona de Estadísitica e Informática. (setiembre de 2017). Caracterísiticas de las Empresas del Emporio Comercial de Gamarra, 2016. Obtenido de www.inei.gob.pe: https://www.inei.gob.pe/media/MenuRecursivo/publicaciones_digitales/Est/Lib1463/libro.pdf

Montuschi, L. (1993). De la retórica del dumping a la práctica del antidumping. (R. I. Plata, Ed.) Económica, 39(1, 2), 47 - 85. Obtenido de http://sedici.unlp.edu.ar/handle/10915/8849

OMC. (s.f.). Las medidas comerciales correctivas y la OMC. Ginebra, Suiza.

Quispe Medina, R. A.-0.-1.-3. (23 de Noviembre de 2017). La elusión de derechos antidumping y compensatorios en el Perú durante el periodo 2003 - 2016 y sus efectos económicos y comerciales sobre la industria nacional. (Tesis de maestría, Universidad Peruana de Ciencias 
Aplicadas). Lima, Perú. Obtenido de https://repositorioacademico.upc.edu.pe/bitstream/handle/10757/622775/Quispe_mr.pdf?se quence $=5$ \&isAllowed $=y$

Ramírez Estrella, A. (2018). Los derechos antidumping como medidas aplicadas para corregir las distorsiones en el mercado. (Tesis de grado), 36. (PUCP, Ed.) Lima, Perú. Obtenido de http://tesis.pucp.edu.pe/repositorio/handle/123456789/11773

Resolución 0293-2015/SDC-INDECOPI. (06 de Junio de 2015). Revoca Derechos Antidumping Definitivos a Prendas de Vestir de China. El Peruano. Lima, Perú: Tribunal de la Competencia y de la Propiedad Intelectual de INDECOPI. Obtenido de https://busquedas.elperuano.pe/normaslegales/se-revoca-la-resolucion-n-297-2013cfdindecopi-del-6-de-di-resolucion-n-293-2015sdc-indecopi-1246386-1/

Resolución 297-2013/CFD-INDECOPI. (22 de Diciembre de 2013). Se Aplican Derechos Antidumping Definitivos a Prendas de Vestir de China. El Peruano. LIma, Perú: Comisión de Fiscalización de Dumping $y$ Subsidios de INDECOPI. Obtenido de https://busquedas.elperuano.pe/download/url/se-aplican-derechos-antidumping-definitivossobre-las-import-resolucion-n-297-2013cfd-indecopi-1030498-1

Rodríguez Fernández, M. (1998). El régimen jurídico de los derechos antidumping en la Comunidad Europea. (Tesis de maestría, Universidad de Oviedo), 604. Oviedo, España. Obtenido de https://www.educacion.gob.es/teseo/mostrarRef.do?ref=197538

Zenere, Y. R. (23 de Marzo de 2013). Instrumentos de Defensa Comercial: Derechos Antidumping en Argentina. RECORDIP, 1(1), $12 . \quad$ Obtenido de https://revistas.unc.edu.ar/index.php/recordip/article/view/4773/4569 